\title{
Facing COVID 19 pandemic: experience from a clinical microbiology laboratory in Spain
}

\begin{abstract}
Objectives: To calculate the turnaround time of results to be able in the electronic medical record. Postulate some available tools regarding laboratory management to assume full response in an increasing demand scenario.

Materials and methods: Retrospective analysis of all samples reaching the lab since February 17 until May 10 was performed using LIS. Records of personnel management and equipment delivery were consulted. Time to results was measured as the difference in hours between time of analytic request and the date of result upload to the electronic medical record.

Results: Time to result started at 24 hours and continually decreased over time reaching stability on week 10 around 6.5 hours. Active measurements taken fall into groups: personnel management, Laboratory schedules and technical capacity.

Conclusion: Adoption of an uninterrupted sample processing method (24/7) and the implementation of high throughput systems are the best options for increasing results performance, where other measurements like redistributing and re train personnel would be more successfully implemented.
\end{abstract}

Keywords: clinical laboratory services,SARS-CoV-2,medical laboratory personnel, clinical laboratory techniques, health policy, COVID-19
Volume 7 Issue 6 - 2020

\author{
Tristancho-Baro Al,' Egido P,' Ortega D,' \\ Mormeneo-Bayo S,' Rezusta $A^{1,2}$ \\ 'Department of Microbiology, Miguel Servet University Hospital, \\ Spain \\ 2IIS Aragón, Zaragoza, Spain
}

Correspondence: Alexander Tristancho Baró, Department of Clinical Microbiology, Miguel Servet University Hospital Zaragoza, Spain, Email alexander.tistanchol@gmail.com

Received: October 21, 2020 | Published: November 02, 2020

\section{Introduction}

On December 31 2019, a cluster of patients with pneumonia of unknown origin in Wuhan (Hubei, China), was notified to WHO local authorities. By January a novel coronavirus named SARSCoV-2 (initially 2019-nCov) was linked as etiological cause. ${ }^{1}$ On March 11 and with reported cases in all five continents pandemic was announced. Two days later, being over 65000 cases outside China, Europe became the epicenter of the pandemic. ${ }^{2}$ Coronaviruses are enveloped, positive-sense single-stranded RNA viruses with a nucleopcapsid and a particular surface protein involved in binding and fusion with host cells called "spike" protein ${ }^{3}$ that gives it a crown (or coroa in latin) aspect on electron microcopy. ${ }^{4} \mathrm{SARS}-\mathrm{CoV}-2$ belongs to the subgenera Sarbecovirus of the Betacoronaviruses which includes SARS-CoV and other less pathogenic human endemic viruses that affect mainly the respiratory and gastrointestinal tract. COVID-19 is the term used to describe the disease caused by SARS-CoV- $2 .{ }^{5}$ Most common symptoms include fever, cough, variable degrees of respiratory distress and less prevalent gastrointestinal and olfactory disturbances. Diffuse pulmonary infiltrate, lymphopenia, elevated C-reactive protein and other markers of inflammation and coagulation are described. ${ }^{6}$ Confirmatory diagnosis is made by viral RNA detection using reverse transcription quantitative polymerase chain reaction (RT-qPCR) from upper or lower respiratory tract samples. Antibody detection may play a role in some cases. ${ }^{7}$ Worldwide mortality rate is $6,3 \%$; however at $27^{\text {th }}$ may is $12,2 \%$ in Spain, mortality rate is also higher in other countries. ${ }^{8}$ Prognosis seems to be worst in patients over 70 years old or comorbidities like hypertension or diabetes ${ }^{9}$ and no specific antiviral treatment or vaccination is available at the time; nevertheless several treatment regiments aimed to decreased viral load and inflammatory response are being approved and research is ongoing..$^{10}$ Microbiology laboratory plays a crucial role in facing COVID-19 pandemic.

\section{Objectives}

a. To stablish the total number of samples reaching the lab and its processing results.

b. To calculate the turnaround time of results to be able for clinical decisions.

c. Postulate some available tools regarding laboratory management to assume full response in an increasing demand scenario, maintaining regular work and reducing staff risk of infection.

d. Describe a particular perspective to deal with COVID-19 pandemic diagnostic process from a Clinical Microbiology point of view.

\section{Material and methods}

Retrospective analysis of all samples reaching the lab since February 17 until May 10 was performed using LIS of the Miguel Servet University Hospital in Zaragoza, Spain. Main variables measured were number of samples by week, interpretation and time to result upload to electronic medical record in hours. Weeks were numbered since the first sample reached the lab in February 17 until May 10. Number of samples are expressed by week.

Samples analyzed consisted of upper and lower respiratory tract, mainly nasopharyngeal (NPS) and oropharyngeal (OPS) swabs in viral transport media when available. Universal lysis buffer were usedprior to extraction and amplification. RNA extraction was performed mainly by magLEADtm (PSS Co., Ltd., Matsudo-city, Japan) and MicrolabSTARlet (Hamilton Company, Reno, US). For RNA targeting, two different protocols were used according to the selected method for extraction and availability. VIASURE SARSCoV-2 Real Time PCR Detection Kit (CerTest BIOTEC, Zaragoza, Spain) and Allplex ${ }^{\text {TM }}$ 2019-nCoV Assay(Seegen, Seul, Korea). CFX96 
Real-Time PCR Detection System (Bio-Rad, Hercules, California, US) was used as the amplification platform. High throughput system COBAS 6800tm (Roche, Basel, Switzerland) encompasses RNA extraction and RT-qPCR in a closed system. Possible interpretations for tests were: Positive, Negative, Non-Conclusive, Inhibited and unprocessed sample. Duplicated samples, lack of unequivocal identification and other Pre-Analytic issues were not suitable for analysis. Analysis interpretation depended on the equipment used to process it. Interpretation criteria was in accordance with published protocols. ${ }^{10,11}$ Time to results was measured as the difference in hours between time of analytic request and the date of result upload to the electronic medical record and expressed as weekly average.Records of personnel management and equipment delivery were consulted to find the time at which every measure was taken and correlate it to other variables.

\section{Results}

A total of 18236 samples were received in the lab within the studied period, of which $18110(99.31 \%)$ were suitable for processing. $3389(18.5 \%)$ positive results were informed, although relative number varies at any given week, reaching its highest on weeks 5 and 6. Samples non-suitable for processing decreased over time as well as inhibited ones in a more discreetly manner, both with relative low frequency. Figure 1 summarizes results in time.Time to result started at 24 hours and continually decreased over time reaching stability on week 10 around 6.5 hours.Although measurements to deal with the outbreak, both in terms of sample management and reducing staff risk of infection, began even before the first case were reported and kept active and evolving during the entire period, the major ones can be placed at different points.Active measurements were taken at weeks $3,4,5,6,9$ and 10 and can be divided into 3 groups: personnel management, Laboratory schedules and technical capacity (Table 1). Implemented in that order respectively, being the major changes in technical capacity adopted the last, at week 9 and 10 . These measurements included hiring new staff, "Recovering" personnel trained in molecular biology diagnostics, redistributing laboratory staff to prioritized essential work, creating specialized retrained "COVID" teams to deal with COVID-19 diagnosis process, slipping workers schedules, implement uninterrupted (24/7) work system for COVID-19 diagnosis, enable high throughput LIS integrated systems and acquire lysis buffer media for primary samples. The time for active measurements, number of samples ant time to result is presented in Figure 2. Seven lab workers $(8.65 \%$ of all staff) were infected and required isolation for a variable time (14-30 days). All presented with mild symptoms and recovered successfully. Particularly, neither of them manipulated primary samples. None of the personnel designated to "COVID teams" were infected.

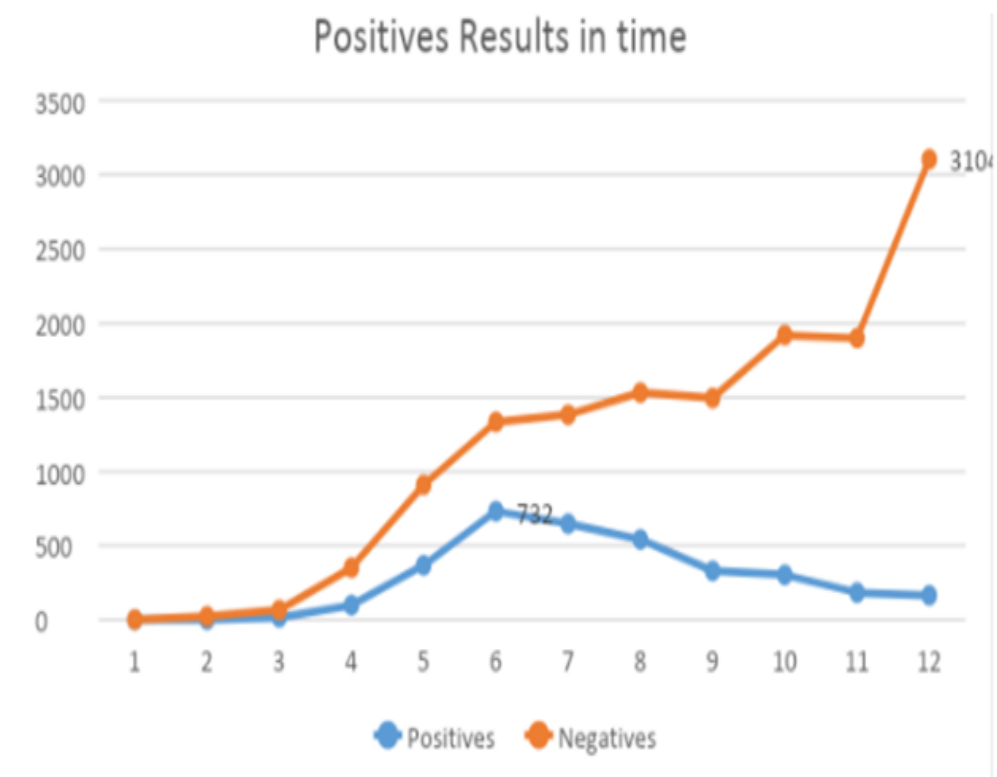

Figure I PCR results in time.

Table I Measurements adopted in the lab to improve performance

\begin{tabular}{ll}
\hline Action & Main resource area \\
\hline Hiring new staff & Personnel management \\
"Recovering" personnel & \\
Redistributing previous staff & \\
Creating "COVID work-teams" & \\
Slipping workers schedules & Laboratory way of function and schedules \\
Allow uninterrupted (24/7) work system & \\
Enable high throughput LIS integrated systems & Technical capacity \\
Acquire Lysis buffer media for primary samples & \\
\hline
\end{tabular}




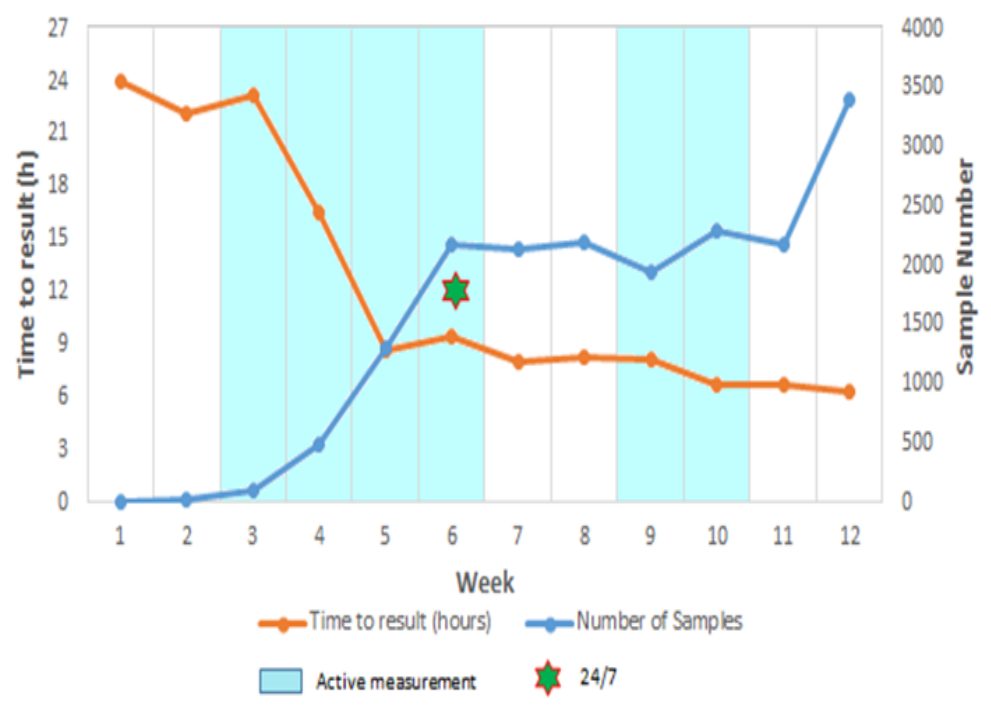

Figure 2 Number of samples, time to results and measurements implemented in time.

\section{Discussion}

The Microbiology Laboratory plays an essential role when facing emerging infectious diseases, offering support in diagnosis, staging, prognostication, therapeutic monitoring, epidemiologic surveillance and research areas, ${ }^{12}$ which in turn helps public health and hospitals decision process. Nevertheless, numerous pre-analytic, analytic and post-analytic issues need to be addressed to ensure safety, quality and timely results. ${ }^{13}$ Changes from baseline work to face COVID-19 pandemic has been necessary in almost every hospital department around the globe and some of those experiences and lessons are published, ${ }^{14-16}$ emphasizing the crucial necessity to adapt emergency policies in order to achieve the better possible outcome; especially when an increasing demand in services is a fact.The acute increase in COVID-19 diagnosis queries (as well as positive results) was expected in accordance to the outbreak national evolution; nevertheless active measurements needed to take place in order to comply with healthcare assistance. Remarkably, the time to results decreased week by week showing the importance and impact of those measures; specially the implementation of uninterrupted COVID-19 diagnosis work in week 6 , as sample number increased by a factor of 4.5 but time to results deceased by $75 \%$ in roughly fifteen days. At present time, RT-qPCR is the gold standard for COVID-19 diagnosis; $;{ }^{12}$ nevertheless, this technique is performed with new and rapidly developed commercial kits, influencing overall process and results interpretation. It is advisable to mention the scope of the test and the most likely variables who affect it. ${ }^{13}$ Although seven lab workers were infected and had to keep isolation for a variable period of time, slipping workers schedules could prevent further cases by avoiding unnecessary contact. Preliminary investigations points to a community rather than laboratory or nosocomial infection.

\section{Conclusion}

The COVID-19 pandemic has had a direct impact in the practical totality of healthcare institutions, generating uncertainty and demanding from all of us the most stoic of attitudes to cope with it for the benefit of our patients and society. Clinical Microbiology department main objective is clear: to develop an optimal circuit that enable an optimal and trustable COVID-19 diagnosis results, so fully collaborative and synergistic work of all health personnel is essential to achieve results with clinical, epidemiological and social impact. Possibly the adoption of an uninterrupted sample processing method (24/7) and the implementation of high throughput systems are the best options for increasing results performance, although it could not be feasible in all laboratories, where other measurements like redistributing and re train personnel would be more successfully implemented.

\section{Ethical approval and informed consent}

Being retrospective in nature and without any potential risk for subjects, no ethics committee submission was necessary neither inform consent.

\section{Acknowledgments}

Special thanks to the Microbiology Laboratory of the Miguel Servet University Hospital.

\section{Conflicts of interest}

No potential conflict of interest was reported by the authors.

\section{Funding}

The present work did not received funds of any kind.

\section{References}

1. Wang L, Wang Y, Ye D, et al. Review of the 2019 novel coronavirus (SARS-CoV-2) based on current evidence. Int J Antimicrob Agents. 2020;105948.

2. Coronavirus disease (COVID-19).

3. Khan S, Siddique R, Shereen MA, et al. Emergence of a Novel Coronavirus, Severe Acute Respiratory Syndrome Coronavirus 2: Biology and Therapeutic Options. J Clin Microbiol. 2020;58(5):e00187-220.

4. Di Gennaro F, Pizzol D, Marotta C, et al. Coronavirus Diseases (COVID-19) Current Status and Future Perspectives: A Narrative Review. Int J Environ Res Public Health. 2020;17(8):2690.

5. He F, Deng Y, Li W. Coronavirus disease 2019: What we know? J Med Virol. 2020;92(7):719-725. 
6. Rodriguez-Morales AJ, Cardona-Ospina JA, Gutiérrez-Ocampo E, et al. Clinical, laboratory and imaging features of COVID-19: A systematic review and meta-analysis. Travel Med Infect Dis. 2020;34:101623.

7. Tang YW, Schmitz JE, Persing DH, et al. Laboratory Diagnosis of COVID-19: Current Issues and Challenges. J Clin Microbiol. 2020;58(6):e00512-520.

8. World Health Organization. Coronavirus Disease (COVID-19) Dashboard.

9. Jin Y, Yang H, Ji W, et al. Virology, Epidemiology, Pathogenesis, and Control of COVID-19. Viruses. 2020;12(4):372.

10. Corman VM, Landt O, Kaiser M, et al. Detection of 2019 novel coronavirus (2019-nCoV) by real-time RT-PCR. Euro Surveill. 2020;25(3):2000045.

11. Liu X, Feng J, Zhang Q, et al. Analytical comparisons of SARS-COV-2 detection by qRT-PCR and ddPCR with multiple primer/probe sets. Emerg Microbes Infect. 2020;1-12.
12. Lippi G, Plebani M. The critical role of laboratory medicine during coronavirus disease 2019 (COVID-19) and other viral outbreaks Clin Chem Lab Med. 2020

13. Lippi G, Simundic AM, Plebani M. Potential preanalytical and analytical vulnerabilities in the laboratory diagnosis of coronavirus disease 2019 (COVID-19). Clin Chem Lab Med. 2020.

14. Tan SS, Yan B, Saw S, et al. Practical laboratory considerations amidst the COVID-19 outbreak: early experience from Singapore. J Clin Pathol. 2020 .

15. Pambuccian SE. The COVID-19 pandemic: implications for the cytology laboratory. J Am Soc Cytopathol. 2020;9(3):202-211.

16. Lam JCM, Moshi GB, Ang SH, et al. Management of COVID-19-related paediatric blood samples in a clinical haematology laboratory. $\mathrm{Br}$ Haematol. 2020;189(5):848-851. 